\title{
Limit Cycles of Quadratic Systems with a Perturbed Weak Focus of Order 3 and a Saddle Equilibrium at Infinity.
}

\author{
G.A. Leonov, N.V. Kuznetsov \\ Draft $^{1} 2$
}

\begin{abstract}
In this paper, we develop a modification of the method of asymptotic integration of Liénard systems and propose an analytical-numerical procedure making it possible to extend the domain of coefficients corresponding to the existence of a limit cycle in a quadratic system with a weak focus of order 3 (for which small perturbations of coefficients yield four limit cycles).
\end{abstract}

Keywords: Limit cycle, 16th Hilbert problem, quadratic system

\section{INTRODUCTION: quadratic systems with weak focus of third order}

After the discovery of the existence of four cycles in quadratic systems with a perturbed weak focus of order 3 (1), the problem of completely describing the domain of existence of such cycles in parameter spaces of quadratic systems has naturally arisen. This problem was considered in $(2 ; 3 ; 4 ; 5 ; 6 ; 7)$.

In this paper, we develop a modification of the method of asymptotic integration of Liénard systems (3) and propose an analytical-numerical procedure making it possible to extend the domain of coefficients corresponding to the existence of a limit cycle in a quadratic system with a weak focus of order 3 (for which small perturbations of coefficients yield four limit cycles $(9)$ ), which was obtained analytically in $(1 ; 2 ; 3 ; 7 ; 8)$.

Consider the special class of Liénard equations obtained by a reduction of the nontrivial quadratic system with zero equilibrium

$$
\begin{aligned}
& \dot{x}=a_{1} x^{2}+b_{1} x y+c_{1} y^{2}+\alpha_{1} x+\beta_{1} y, \\
& \dot{y}=a_{2} x^{2}+b_{2} x y+c_{2} y^{2}+\alpha_{2} x+\beta_{2} y,
\end{aligned}
$$

where $c_{1}=0$ (this can always be achieved by making the change $x \rightarrow(x+\nu y)$ for $a_{1} \neq 0$ or by changing notation as $x \leftrightarrow y)$ and $b_{1} \beta_{1} \neq 0$. The straight line $b_{1} x+\beta_{1}=0$ is a transversal line of system (1.1), which can be transformed into a system with $b_{1}=\beta_{1}=1(5 ; 6 ; 3)$ and reduced to the Liénard equation

$$
\ddot{x}+f(x) \dot{x}+g(x)=0
$$

with functions $f$ and $g$ of the form

$$
\begin{aligned}
& f(x)=\left(A x^{2}+B x+C\right)|x+1|^{q-2}, \\
& g(x)=\left(C_{1} x^{4}+C_{2} x^{3}+C_{3} x^{2}+C_{4} x+C_{5}\right) \frac{|x+1|^{2 q}}{(x+1)^{3}} .
\end{aligned}
$$

It is well known that, in a quadratic system, up to three small cycles around one equilibrium point (a weak focus) can be obtained provided that the first two Lyapunov quantities vanish and the third does not

\footnotetext{
${ }^{1}$ Nikolay V. Kuznetsov, nkuznetsov239 at gmail.com (correspondence author)

${ }^{2} \mathrm{PDF}$ slides http://www.math.spbu.ru/user/nk/PDF/Limit-cycles-Focus-values-Lyapunov-quantity-16th-Hilbert.pdf
} 
vanish (9). In this case, reducing the system to the Liénard equation, we obtain the following relations for the coefficients of functions $(1.3)(5 ; 6)$

$$
\begin{aligned}
& A=\frac{2}{5} B(q+2) \\
& C_{1}=(q+3) \frac{B^{2}}{25}-\frac{(1+3 q)}{5} \\
& C_{2}=\left(15(1-2 q)+3 B^{2}\right) \frac{1}{25} \\
& C_{3}=\frac{3(3-q)}{5} \\
& C_{4}=1, C_{5}=0 .
\end{aligned}
$$

In $(4 ; 5 ; 6 ; 10)$, the Lyapunov quantities for Eq.(1.2) with coefficients (1.4) were calculated by the direct method, and at the zero equilibrium point, an expression for the third Lyapunov quantity was obtained; this expression has the form

$$
\mathrm{L}_{3}=-\frac{\pi B(q+2)(3 q+1)\left[5(q+1)(2 q-1)^{2}+B^{2}(q-3)\right]}{20000}
$$

The domain of parameters $(B, q)$ corresponding to the existence of a large limit cycle on the left of the line of discontinuity $x=-1$ for such an equation (and the corresponding quadratic system) was analytically estimated in (3) as

$$
B^{2}<-5(q+1)(3 q+1), B \neq 0
$$

This domain extends the class of quadratic systems considered by Shi in (1).

To further study this domain, we apply numerical procedures and the method of asymptotic integration. Making the change of variables $x=-w-2$ (this is the symmetry with respect to the line of discontinuity $x=-1)$ in (1.2) and collecting the coefficients of powers of $(1+w)$, we obtain the equation

$$
\begin{aligned}
& \ddot{w}+f(w) \dot{w}+g(w)=0 \\
& f(w)=\left(\widetilde{A}_{w}(1+w)^{2}+\widetilde{B}_{w}(w+1)+\widetilde{C}_{w}\right)|w+1|^{q-2} \\
& g(w)=\left(\widetilde{C}_{w 1}(1+w)^{4}+\widetilde{C}_{w 2}(1+w)^{3}+\widetilde{C}_{w 3}(1+w)^{2}+\right. \\
& \left.+\widetilde{C}_{w 4}(1+w)+\widetilde{C}_{w 5}\right) \frac{|w+1|^{2 q}}{(w+1)^{3}}
\end{aligned}
$$

By virtue of conditions (1.4), we have

$$
\begin{aligned}
& \widetilde{A}_{w}=\frac{2}{5} B(2+q), \widetilde{B}_{w}=\frac{1}{5} B(3+4 q), \widetilde{C}_{w}=\frac{1}{5} B(-1+2 q), \\
& \widetilde{C}_{w 1}=\frac{1}{25}\left((3+q) B^{2}-15 q-5\right), \widetilde{C}_{w 2}=\frac{1}{25}\left((4 q+9) B^{2}-35-30 q\right), \\
& \widetilde{C}_{w 3}=\frac{1}{25}\left((6 q+9) B^{2}-30-15 q\right), \widetilde{C}_{w 4}=\frac{1}{25} B^{2}(3+4 q), \\
& \widetilde{C}_{w 5}=\frac{1}{25} B^{2} q .
\end{aligned}
$$

Note that, according to (1.4), to study the existence of cycles, it suffices to consider $B$ of only one sign. In what follows, we assume that

$$
B<0 \text {. }
$$




\section{ASYMPTOTIC INTEGRATION OF THE LIÉNARD EQUA- TION}

Let us apply the method of asymptotic integration to the Liénard equation (3). For $y=\dot{x} \neq 0$, we rewrite (1.2) at the first-order equation

$$
\frac{d y}{d x}+f(x)+\frac{g(x)}{y}=0
$$

and assume that, on the interval $(a, b)$,

$$
\begin{aligned}
& f(x)=f_{0}+f_{1}(x), \quad f_{0} \neq 0, \lim _{x \rightarrow a} f_{1}(x)=0, \\
& g(x)=g_{0}+g_{2}(x) \text { or } \quad g(x)=g_{1} x+g_{2}(x) x, \quad \lim _{x \rightarrow a} g_{2}(x)=0 .
\end{aligned}
$$

Consider the approximation

$$
\frac{d y_{l i n}}{d x}+f_{0}+\frac{g_{0}+g_{1} x}{y_{l i n}}=0
$$

of Eq.(2.8) on the interval $(a, b)$ and its solution

$$
\begin{array}{lll}
y_{\text {lin }}(x)=k, & k=-\frac{g_{0}}{f_{0}}, & g_{0} \neq 0, g_{1}=0 . \\
y_{\text {lin }}(x)=k x, & k=-\frac{f_{0}}{2} \pm \sqrt{\frac{f_{0}^{2}}{4}-g_{1}}, & g_{0}=0, g_{1} \neq 0
\end{array}
$$

By virtue of (2.9), the solution $y_{\text {lin }}(x)$ thus obtained is an approximation to the solution $y(x)$ of nonlinear equation (2.8) with initial data $\left(x_{0}, y_{\text {lin }}\left(x_{0}\right)\right)$ for $x_{0}$ close to $a$.

To reduce the functions in Eq.(1.2) to the form (2.9), we make the change variables $x=D(z)$, where $D(z):\left(a_{z}, b_{z}\right) \rightarrow(a, b)$ is a smooth function. Introducing the notation

$$
\widetilde{f}(z)=\frac{d D(z)}{d z} f(D(z)), \widetilde{g}(z)=\frac{d D(z)}{d z} g(D(z)),
$$

we obtain the equation

$$
\frac{d y}{d z}+\widetilde{f}(z)+\frac{\widetilde{g}(z)}{y}=0, \quad z \in\left(a_{z}, b_{z}\right) .
$$

Applying this method to Eq.(2.8) with functions of the form (2.9), we can construct special separating solutions on the phase portrait and perform effective procedures of limit cycles localization.

\section{CONSTRUCTION OF SEPARATING SOLUTIONS AND AN ANALYSIS OF THE EXISTENCE OF "NORMAL" LIMIT CYCLES}

In what follows, we consider $q \in(-2,0)$ and make various changes of variables to construct separating solutions of Eq.(1.7). It is easy to show (3) that the function $g(w)$ has only one zero (i.e., the equation has only one equilibrium point) on the right of the discontinuity line $w=-1$.

Suppose that $q \in(-2,-1)$. For $w \in(0,+\infty)$, we make the change

$$
w=D(z)=z^{\frac{1}{q+1}}-1:(0,1) \rightarrow(0,+\infty),
$$




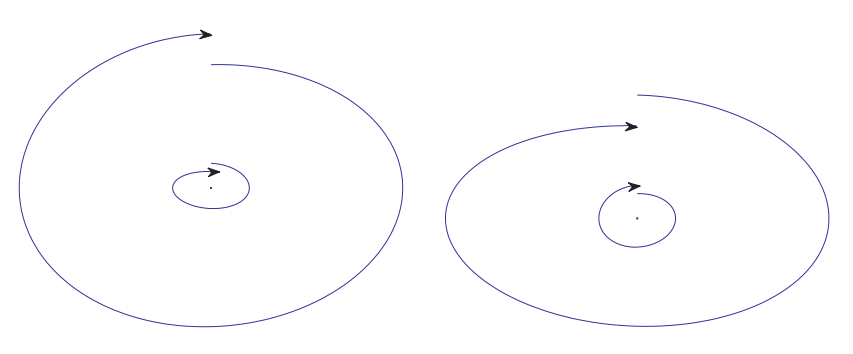

Figure 1: Licalization of an unstable cycle (a) and a stable cycle (b)

in Eq.(1.7); the function $D(z)$ monotonically decreases. We obtain

$$
\begin{aligned}
& \widetilde{f}(z)=\frac{1}{(q+1)}\left(\widetilde{A}_{w}+\widetilde{B}_{w} z^{-\frac{1}{q+1}}+\widetilde{C}_{w} z^{-\frac{2}{q+1}}\right) \\
& \widetilde{g}(z)=\frac{1}{(q+1)} z\left(\widetilde{C}_{w 1}+z^{-\frac{1}{q+1}} \widetilde{C}_{w 2}+z^{-\frac{2}{q+1}} \widetilde{C}_{w 3}+\right. \\
& \left.+z^{-\frac{3}{q+1}} \widetilde{C}_{w 4}+z^{-\frac{4}{q+1}} \widetilde{C}_{w 5}\right)
\end{aligned}
$$

According to (2.9), we have

$$
\widetilde{f}_{0}=\frac{\widetilde{A}_{w}}{(q+1)}, \quad \widetilde{g}_{1}=\frac{\widetilde{C}_{w 1}}{(q+1)}<0
$$

The eigenvalues

$$
k_{1,2}=-\frac{\tilde{f}_{0}}{2} \pm \sqrt{\frac{\tilde{f}_{0}^{2}}{4}-\widetilde{g}_{1}}
$$

correspond to the two separatrices $y_{\text {lin, } 1}(z) \quad=\quad k_{1} z \quad$ and $y_{l i n, 2}(z)=k_{2} z$. The mapping of the separatrices to the phase plane $(w=D(z), y)$ yields two trajectories on different sides of the line $y=0$, which start from the line $w=0$ and intersect in $w=+\infty$, which is the image of the point $z=0$.

To determine the corresponding separating solutions $y_{1}(z)$ and $y_{2}(z)$ of nonlinear equation (2.11) with functions $\widetilde{f}(z)$ and $\widetilde{g}(z)$, we take initial data on the lines $y_{\text {lin }, 1}(z)=k_{1} z$ and $y_{\text {lin }, 2}(z)=k_{2} z$ sufficiently close to the point $z=0$ and numerically determine solutions $y_{1}\left(z, z_{0}, k_{1} z_{0}\right)$ and $y_{2}\left(z, z_{0}, k_{2} z_{0}\right)$.

We map the separating solutions $y_{1}(z), y_{2}(z)$ thus obtained to the phase plane $(w=D(z), y)$ and consider the resulting trajectories $y_{1}(w)$ and $y_{2}(w)$. Applying numerical modeling, we find parameters $(B, q)$ for which the equilibrium state is locally stable (unstable) and the trajectory $T$ from the endpoint (corresponding to $w=0)$ of the lower separating solution $y_{2}(w)$ passes the equilibrium point from the left, makes a half-turn, and go to the right above (below) the upper separating solution $y_{1}(w)$. This corresponds to the existence on the phase portrait of an exterior "unwinding" (Fig. 1, a) or an exterior "winding" (Fig. 1, b) around a locally stable or an unstable equilibrium, respectively, which ensures the presence of a limit cycles.

Applying this technique to $q \in[-1,0)$, we can construct two separating solutions $y_{1}(w)$ and $y_{2}(w)$, which start at the line $w=0$ and tend to the line of discontinuity $w=-1$ and to $+\infty$, respectively. For this purpose, we use two different changes of the form $w=D(z)$, for mappings from $(1,+\infty)$ to $(0,+\infty)$ and to 
$(-1,0)$. The first mapping has the form

$$
\text { for } q \in(-1,0)\left\{\begin{array}{l}
D(z)=z^{\frac{1}{q+1}}-1:(1,+\infty) \rightarrow(0,+\infty), \\
\widetilde{f}_{0}=\frac{\widetilde{A}_{w}}{(q+1)}<0, \widetilde{g}_{0}=0, \widetilde{g}_{1}=\frac{\widetilde{C}_{w 1}}{(q+1)}, \\
k_{1}=-\frac{\widetilde{f}_{0}}{2}-\sqrt{\frac{\widetilde{f}_{0}^{2}}{4}-\widetilde{g}_{1},} \\
\text { for } q=-1 \\
\begin{array}{l}
D(z)=e^{z}-1:(0,+\infty) \rightarrow(0,+\infty), \\
\widetilde{f}_{0}=\widetilde{A}_{w}, \widetilde{g}_{0}=\widetilde{C}_{w 1}, k_{1}=-\frac{\widetilde{f}_{0}}{\widetilde{g}_{0}},
\end{array}
\end{array}\right.
$$

where $D(z)$ monotonically increases monotonically increases (note that, in this case, the negative radicand in the expression for $k_{1}$ corresponds to the condition (1.6) for the existence of a limit cycle). The second mapping has the form

$$
\left\{\begin{array}{c}
D(z)=z^{\frac{1}{q-1}}-1:(1,+\infty) \searrow(-1,0), \\
\widetilde{f}_{0}=\frac{\widetilde{C}_{w}}{(q-1)}<0, \widetilde{g}_{0}=0, \widetilde{g}_{1}=\frac{\widetilde{C}_{w 5}}{(q-1)}, \\
k_{2}=-\frac{\widetilde{f}_{0}}{2}-\sqrt{\frac{\widetilde{f}_{0}^{2}}{4}-\widetilde{g}_{1}}
\end{array}\right.
$$

where $D(z)$ monotonically decreases and the radicand is always positively.

\section{APPLICATION RESULTS: quadratic system with four limit cycles}

The application of the analytical-numerical procedure described above to various parameters $(B, q)$ has made it possible to obtain the domain of parameters (the shaded domain under the ellipse in Fig. 2), corresponding to the existence of a limit cycle. This domain complements the domain $\Omega$ obtained analytically in $(2 ; 3 ; 7)$ (the domain bounded by the ellipse in Fig. 2) and includes examples considered in (11; 12), which reduced to the Liénard equation with parameters $(B=-0.2607, q=-1.03)$ and $(B=-0.2571, q=-1.04)$.

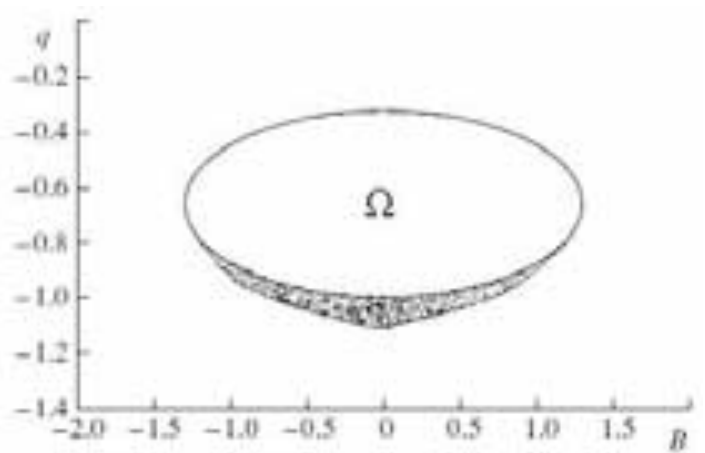

Figure 2: The domain of parameters $(B, q)$ on which small perturbations of coefficients may yield four limit cycles 


\section{References}

[1] Shi S. L. A concrete example of the existence of four limit cycles for plane quadratic systems. Sci. Sinica, 23, 1980, pp. 153-158.

[2] Artes J.C. and Llibre J. Quadratic vector fields with a weak focus of third order, Pub. Math., No 41, 1997, pp. 7-39.

[3] G.A. Leonov, Dokl. Akad. Nauk, 426, 2009, 47-50.

[4] Kuznetsov N.V. Stability and Oscillations of Dynamical Systems: Theory and Applications. Jyväskylä University Printing House, Jyväskylä, 2008. 120 p.

[5] Leonov G. A., Kuznetsov N. V., and Kudryashova E. V. Limit cycles of two-dimensional systems. Calculations, proofs, experiments. Vestnik St.Petersburg University. Mathematics, 41(3), 2008, pp. 216-250.

[6] Kuznetsov N. V. and Leonov G. A. Limit cycles and strange behavior of trajectories in two dimension quadrat Journal of Vibroengineering, 2008, 10(4), pp. 460-467.

[7] Kuznetsov N.V., Leonov G.A. Computation of Lyapunov quantities for Lienard equation, (book chapter in Dynamics and Control of Hybrid Mechanical Systems, editors G. Leonov, H. Nijmeijer, A. Pogromsky, A. Fradkov, World Scientific, 2010), pp. 7-28.

[8] G.A. Leonov, Prikl.Mat. Mekh., 74 (1),2010, pp. 37-73.

[9] N.N. Bautin, Mat. Sb., 30 (1), 1952, pp. 181-196.

[10] G.A. Leonov, N.V. Kuznetsov, E.V. Kudryashova, A Direct Method for Calculating Lyapunov Quantities of Two-Dimensional Dynamical Systems, Proceedings of the Steklov Institute of Mathematics, Suppl. 3, 2010, pp. S1-S8

[11] Cherkas L. A., Artes J.C. and Llibre J., Bul. Acad. Stiinte Rep. Moldova. Mat. 41 (1), 2003, pp. 31-46.

[12] L.A. Cherkas and I.L.Shevtsov, Dokl. Belorus. Gos. Univ. Inform. Radioelektr., No 1, 2004, pp. 115-125. 